\title{
HARMONICS AND INTERHARMONICS Estimation of A Passive Magnetic Fault CURRENT LIMITER USING MORLET WAVELET TRANSFORM
}

\author{
Raju Patwary ${ }^{1}$ and Pratyush Das ${ }^{2}$ \\ ${ }^{1}$ Department of Electrical Engineering, Gargi Memorial Institute of \\ Technology, Balarampur, Baruipur,24Parganas(S) \\ rajpat_2008@yahoo.com \\ ${ }^{2}$ Department of Electrical Engineering, Global Institute of Management and \\ Technology, Krisnagar, Nadia \\ pratyush.85@gmail.com
}

\begin{abstract}
This paper presents the harmonics and interharmonics analysis of a passive magnetic fault current limiter (MFCL). This device limits the current at post fault without affecting the pre fault state of the system. The harmonics and interharmonics estimation of a non-stationary signal generated by MFCL has been investigated using Morlet Wavelet transform and FFT. Continous wavelet transform (CWT) have been applied for estimation of harmonics and interharmonics in MFCL under normal and faulted condition.
\end{abstract}

\section{KEYWORDS}

FCL, Harmonics, Interharmonics, Continuous Wavelet Transform.

\section{INTRODUCTION}

With increase in electricity demand the power system fault level increases. Traditional techniques of fixing fault current have been reported in [1]. But all these techniques suffer from some disadvantages. Magnetic fault current limiter is a novel approach of limiting fault current without affecting the normal state. This helps us to increase the fault level of a system without upgrading the existing switchgear. It needs no sensing and control devices, no additional power supply or any auxiliary winding. Its operation is automatic and instantaneous. In this paper the harmonic and interharmonic estimation of a non-stationary signal in presence of MFCL has been analysed. This investigation is required as many solid-state fault current limiters [5] inject undesirable harmonics in the system, thus affecting the quality of power. The investigation of the harmonics and interharmonics content of a non-stationary signal in presence of MFCL under normal and faulted condition has been estimated based on wavelet transform as wavelets are well suited for the analysis of transient time-varying signals.

\section{Magnetic Fault Current Limiter}

An MFCL consists of a permanent magnet (NdFeB) and two low saturation flux density materials such as a ferrite core as shown in Fig-1. [2] The coils are connected such that any instant of the Rupak Bhattacharyya et al. (Eds) : ACER 2013, pp. 427-436, 2013. @ CS \& IT-CSCP 2013

DOI : $10.5121 / \mathrm{csit} .2013 .3240$ 
a.c cycle, the m.m.f generated by the a.c source and the permanent magnet assist each other in one core and opposes each other in the another and both cores remain saturated below a specific value of applied current.

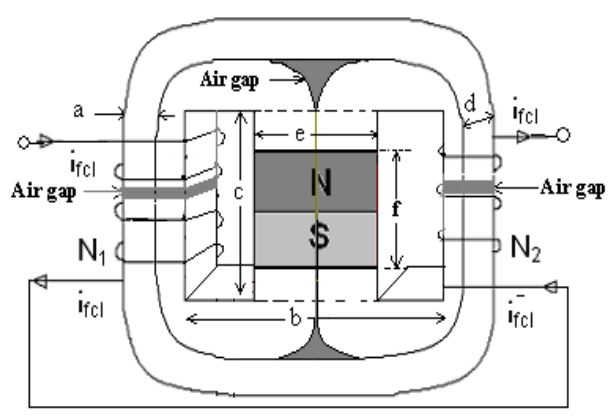

Fig.1 Parallel Biased MFCL

The permanent magnet will bias the core and forces it to operate in saturation. [3, 4] Magnetic current limiter behaves as a variable inductor. Under normal operating condition below the current (maximum nominal current), MFCL offers low saturated inductance $\mathrm{L}_{\mathrm{s}}$. Under fault condition (Max nominal current $<\mathrm{I} \leq$ Max fault current), the inductance offered by the MFCL is the high unsaturated inductance $\mathrm{L}_{\mathrm{u}}$. The design parameters are such that $\mathrm{L}_{\mathrm{u}}>>\mathrm{L}_{\mathrm{s} \text {. }}$ Hence under faulted condition the effective inductive reactance of MFCL is very high, thus limiting the current.

\section{RESPONSE OF MFCL}

A prototype laboratory model of MFCL has been designed with the data shown in Table-1. Software simulation has been carried out to obtain the graphs shown in Figs. 3.

Table-1: Design Data

\begin{tabular}{|c|c|}
\hline Core & $\begin{array}{c}\mathrm{a}=7.5 \mathrm{~mm} ; \mathrm{b}=24 \mathrm{~mm} ; \mathrm{d}=20 \mathrm{~mm} ; \mathrm{c}=48 \mathrm{~mm}, \mu_{\mathrm{rs}}(\text { saturated permeability })=87.6 * \mu_{\mathrm{o}} ; \\
\mu_{\mathrm{ru}}(\text { unsaturated permeability })=12740 * \mu_{\mathrm{o}} ; B_{\mathrm{k}}(\text { Knee flux density })=0.35 \mathrm{~T} ; \\
\text { Ferrite core }\end{array}$ \\
\hline $\begin{array}{r}\text { Permane } \\
\text { Magnet }\end{array}$ & $\begin{aligned} \mathrm{e}=15 \mathrm{~mm} ; \mathrm{f}=5 \mathrm{~mm}, \mu_{\mathrm{ru}}= & 2.4 \times 10^{-6} \mathrm{H} / \mathrm{m}, \mathrm{H}_{\mathrm{c}}\left(\text { coercivity) }=0.8^{*} * 10^{6} \mathrm{~A} / \mathrm{m}, \mathrm{NdFeB}\right. \\
& 2 \text { Neodymium Iron Boron) }\end{aligned}$ \\
\hline Othe & $\begin{array}{l}\text { No. of turns }=100 \text { per limb; } V_{s}=15 \mathrm{~V} \text { r.m.s; } R_{\mathrm{LN}=1} 1^{\prime} \Omega(\text { Normal load resistance) } \\
\qquad R_{\mathrm{LF}}=1.7^{\prime} \Omega \text { (fault resistance) }\end{array}$ \\
\hline
\end{tabular}

Fig.2 shows the simulation circuit of MFCL. Under normal operating condition when switch ' $\mathrm{S}$ ' is open the load across MFCL is $R_{\mathrm{LN}}$. In fault condition, the switch ' $\mathrm{S}$ ' is closed and the load across MFCL is $R_{L N}$ parallel with $R_{L F}$. Fault is created at time $t=0.02$ sec.by closing the switch ' $S$ '.From Fig. 3 it is evident that the fault current is significantly limited by the use of MFCL[4]. 


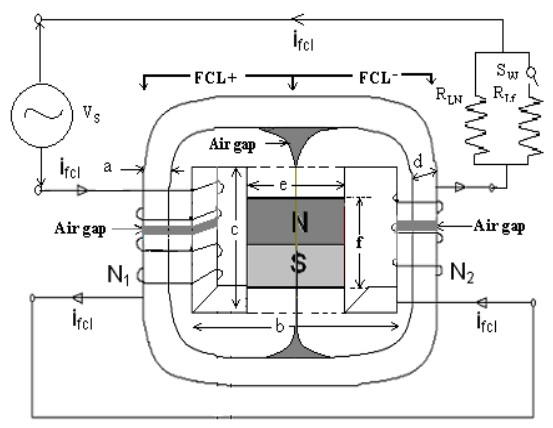

Fig.2 Simulation Circuit of MFCL

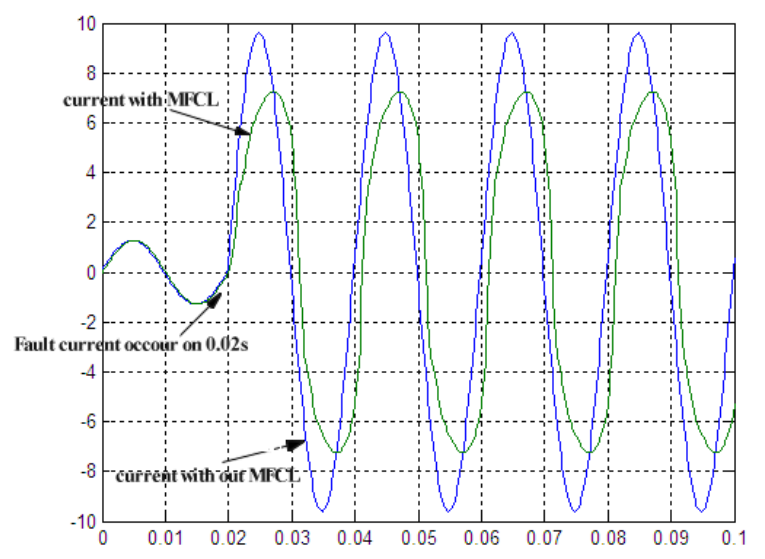

Fig.3 MFCL Current Vs Time

Thus the MFCL limits the fault current significantly and voltage regulation at normal state is also good. It is to be investigated whether the presence of MFCL in a network injects any undesiarable harmonics or interharmonics in the supply system to degrade the quality of power.

\section{HARMONICS AND INTERHARMONICS ANALYSIS}

Traditionally the Fast Fourier Transform (FFT) has been used to analyze harmonics in power system. The FFT is able to give accurate results only if the analyzed waveform fulfills certain conditions [8]. FFT analysis cannot give any inter harmonics estimation, because due to spectral leakage FFT produces enormous errors. The wavelet transform has been introduced for harmonic measurements of power systems. The wavelet transform is capable of accurately extracting the information contained in any distorted waveform with high resolution both in time and frequency. For harmonic measurement purposes, the frequency spectrum should be divided into uniform bands. This can only be realized using the Wavelet Packet Transform (WPT). [6] In this paper the harmonic estimation of MFCL current signal is presented based on discrete wavelet packet transform (DWPT) and continous wavelet transform (CWT). To achieve good time and frequency resolution of a nonstationary signal wavelet is developed.The continous wavelet transform (CWT) of a signal $f(t)$ is given by

$W(a, b)=\int_{t} f(t) \frac{1}{\sqrt{|a|}} \psi\left(\frac{t-b}{a}\right) d t$ 
Where wavelet is denoted by

$\psi_{a, b}(t)=\int_{t} \frac{1}{\sqrt{|a|}} \psi\left(\frac{t-b}{a}\right) d t$

Here ' $a$ ' is dilation or scaling parameter and ' $b$ ' is translation or location parameter.

By choosing $a=a_{0}^{m}, b=\mathrm{n} b_{0}$ and $\mathrm{t}=\mathrm{kT}$ in equation (1), where $\mathrm{T}=1.0$ and $\mathrm{m}, \mathrm{n}, \mathrm{k}$ are integer values. The discrete wavelet transform is given by

$$
D W T(m, n)=\left(\sum_{k} x(k) \psi\left[\left(k-n b_{0}\right) / a_{0}^{m}\right]\right) / a_{0}^{m}-
$$

For computational effeciency $\mathrm{a}_{0}$ and $\mathrm{b}_{0}$ are set to 2 and 1 respectively.Multilevel filter bank is iplimented on equation (3) to get discrete wavelet packet transform

$\psi_{2 n}(t)=2 \sum_{k=0}^{2 N-1} L(k) \psi_{n}(2 t-k)-----------(5)$
$\psi_{2 n+1}(t)=2 \sum_{k=0}^{2 N-1} H(k) \psi_{n}(2 t-k)$----------- (6)

The wavelet packets $\psi_{n}$ are generated from the linear combination of scaled and translated version of mother wavelet $\psi_{1}(t)$ and its scaling function $\psi_{0}(t)$. L and $\mathrm{H}$ is low pass and high pass filter of length $2 \mathrm{~N}$ corresponds to mother wavelet.

In this paper we have used Morlet (morl) mother wavelet, which is compactly supported orthonormal wavelets, making discrete wavelet analysis practicable. These wavelets have no explicit expression except for morl, which is the Morlet wavelet. However, the square modulus of the transfer function of $h$ is explicit and fairly simple. If the polynomial $\mathrm{P}(\mathrm{y})$ can be expressed as

$P(y)=\sum_{k=0}^{N-1} C_{k}^{N-1+k} y^{k}$

Then $\left|m_{0}(w)\right|^{2}=\left(\cos ^{2}\left(\frac{w}{2}\right)\right)^{N} P\left(\sin ^{2}\left(\frac{w}{2}\right)\right)$ here $m_{0}(w)=\frac{1}{\sqrt{2}} \sum_{k=0}^{2 N-1} h_{k} e^{-i k w}$ and $N$ is the number of vanishing moments.

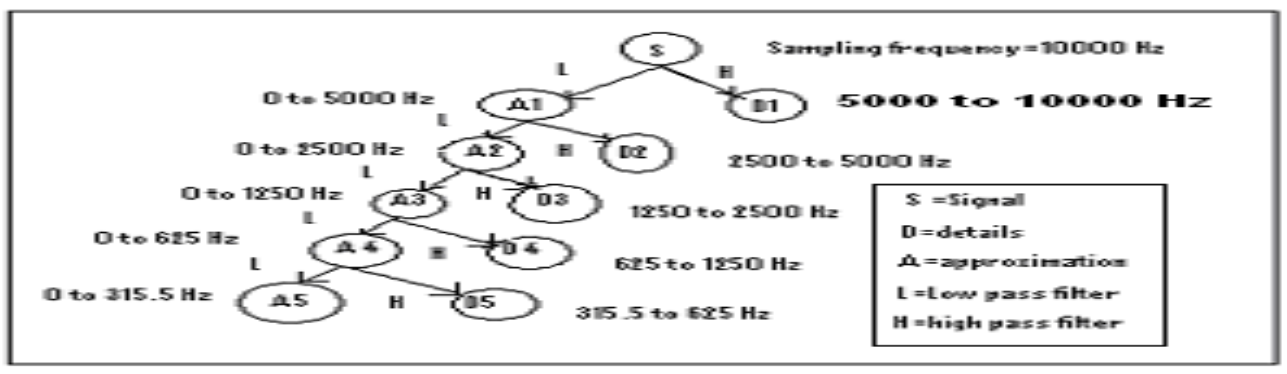

Fig: 4: DWPT Filter bank 


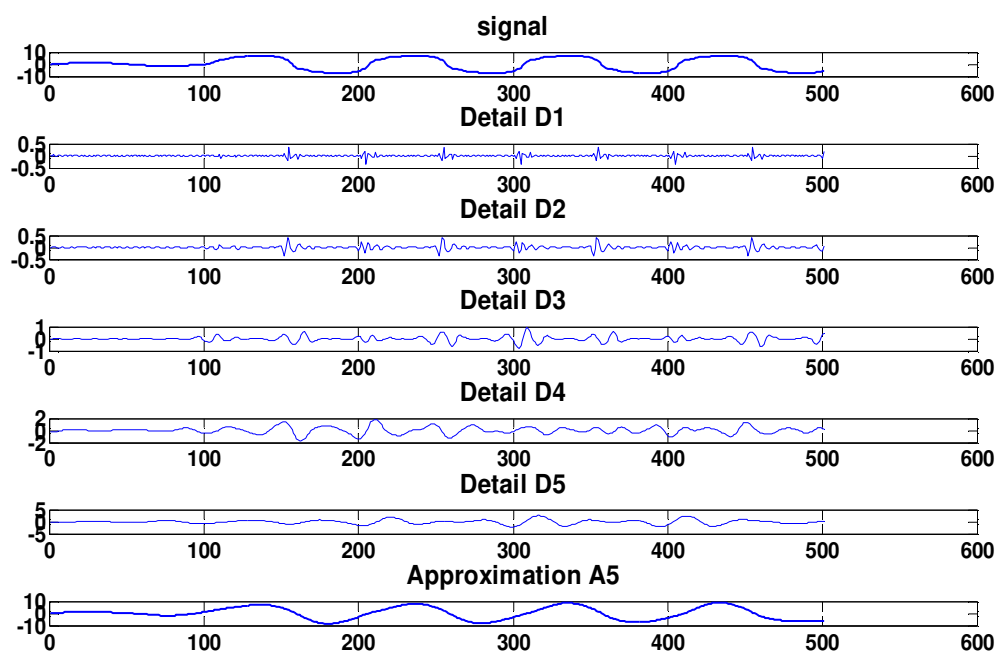

Fig. 5 The first 6 decomposed components of MFCL current

Discrete wavelet packet in this paper shown in Fig 4.The discrete wavelet packet transform gives a uniform band frequency at each level which is very suitable for harmonics and interharmonics studies. After applying the DWPT at level 5 on MFCL current signal, we get the desired details and approximation as shown in Fig. 5. It is evident that at normal condition $(0-0.02 \mathrm{~s}$ or $0-100 \mathrm{hz})$ no significant harmonic is being generated. Harmonics generated mainly in fault condition (0.020.1 sor 0 to $500 \mathrm{hz}$ ). It is observed that at fault condition the magnitude of higher harmonics is very less and the harmonics and interharmonics of high magnitude occur mainly in frequency band of $0-315 \mathrm{~Hz}$ (refer table-2).

Table-2: Sub-band frequency and magnitude

\begin{tabular}{|c|c|c|c|}
\hline \multirow{2}{*}{ Sub- band } & \multirow{2}{*}{ Frequency range (Hz) } & \multicolumn{2}{|c|}{$\begin{array}{c}\text { Magnitude } \\
\text { Range (A) }\end{array}$} \\
\cline { 3 - 4 } & & $\begin{array}{c}\text { Normal } \\
\text { State }\end{array}$ & Faulty state \\
\hline D1 & $5000-10000$ & 0.00 & 0.2 \\
\hline D2 & $2500-5000$ & 0.00 & 0.4 \\
\hline D3 & $1250-2500$ & 0.00 & 0.8 \\
\hline D4 & $625-1250$ & 0.00 & 1.8 \\
\hline D5 & $315.5-625$ & 0.05 & 3.8 \\
\hline A5 & $0-315.5$ & 1.95 & 9.8 \\
\hline
\end{tabular}




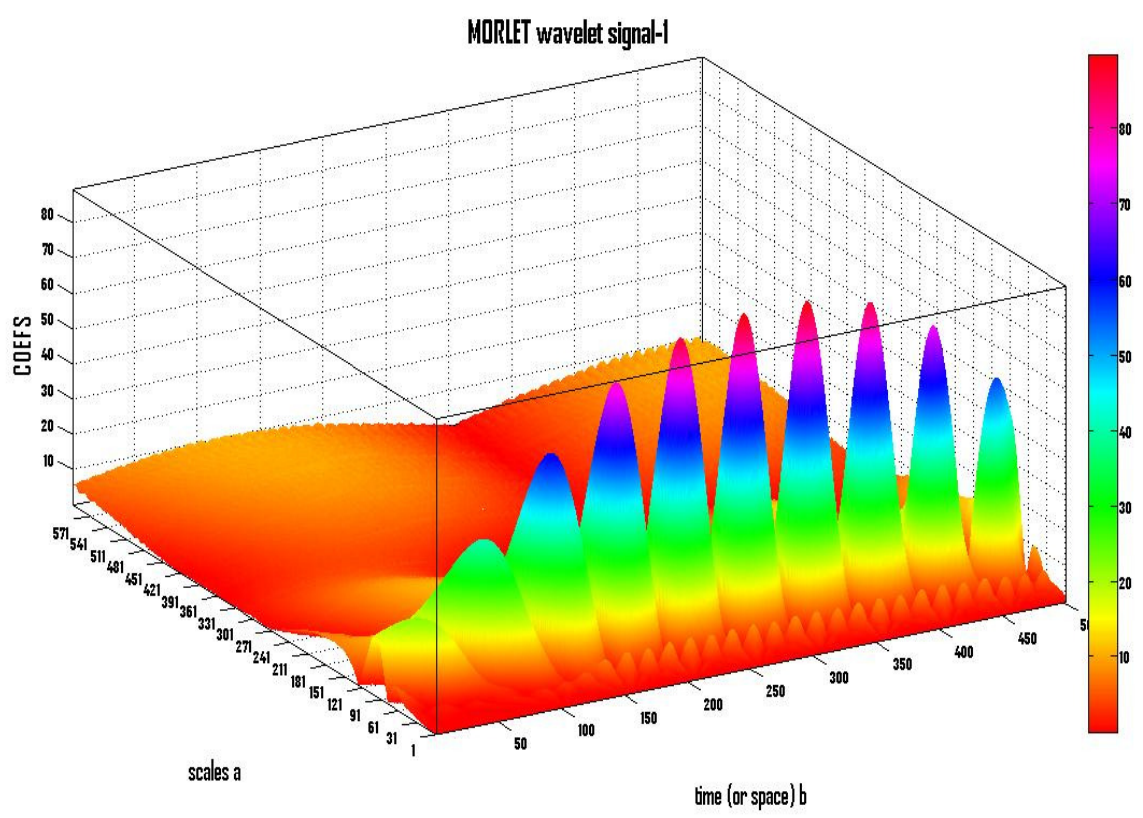

Fig.6 Time-frequency-amplitude plot by CWT

Fig.-6 shows the continuous wavelet transform (CWT) of the current signal of MFCL as shown in Fig- 3 with Morlet mother wavelet. Here we observe the time-freq-magnitude plot of the MFCL current signal. From 0-0.02 is the normal state and 0.02-0.1 is the faulty state. It is clearly observed that the magnitude of fundamental component $(50 \mathrm{~Hz})$ is maximum and the higher order component has very negligible magnitude. There is some amount of sub harmonics components whose magnitude is also very less. Thus CWT supports the conclusion drawn from DWPT.
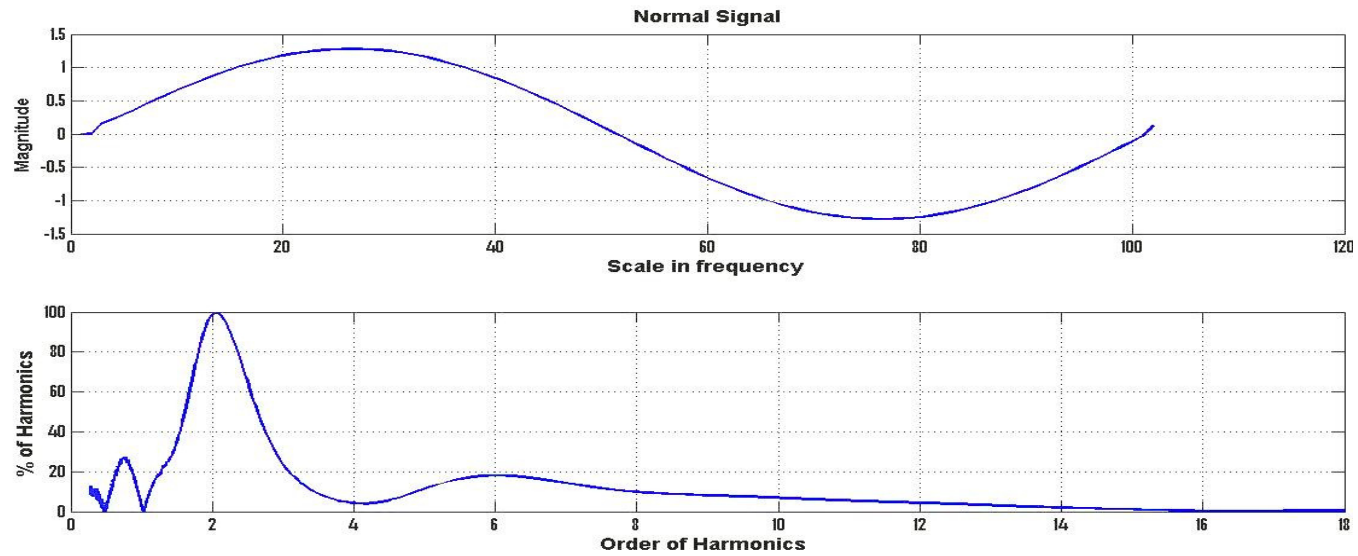

Fig.7 Frequency spectrum of MFCL current at normal state by Wavelet 

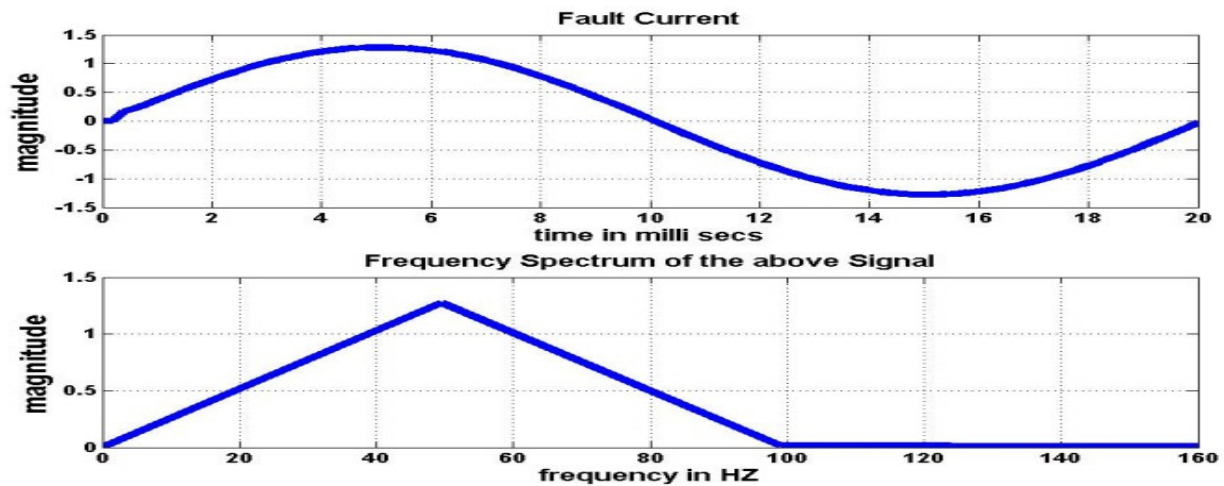

Fig.8 Frequency spectrum of MFCL current at normal state by FFT

To know the exact harmonic and inter harmonic components and their magnitude in the nonstationary signal generated by MFCL wavelet transform and Fast Fourier Transform has been carried out. For frequency spectrum analysis 600 numbers of samples are taken for normal condition and faulted condition as shown in Fig-7 \& 10.

Fig $7 \& 8$ shows the frequency spectrum for MFCL current at normal state by WT and FFT respectively. Here we observe that mainly the $1^{\text {st }}$ harmonic component constitute the MFCL current signal at normal condition. There is almost no significant higher order harmonics in the MFCL current at normal state
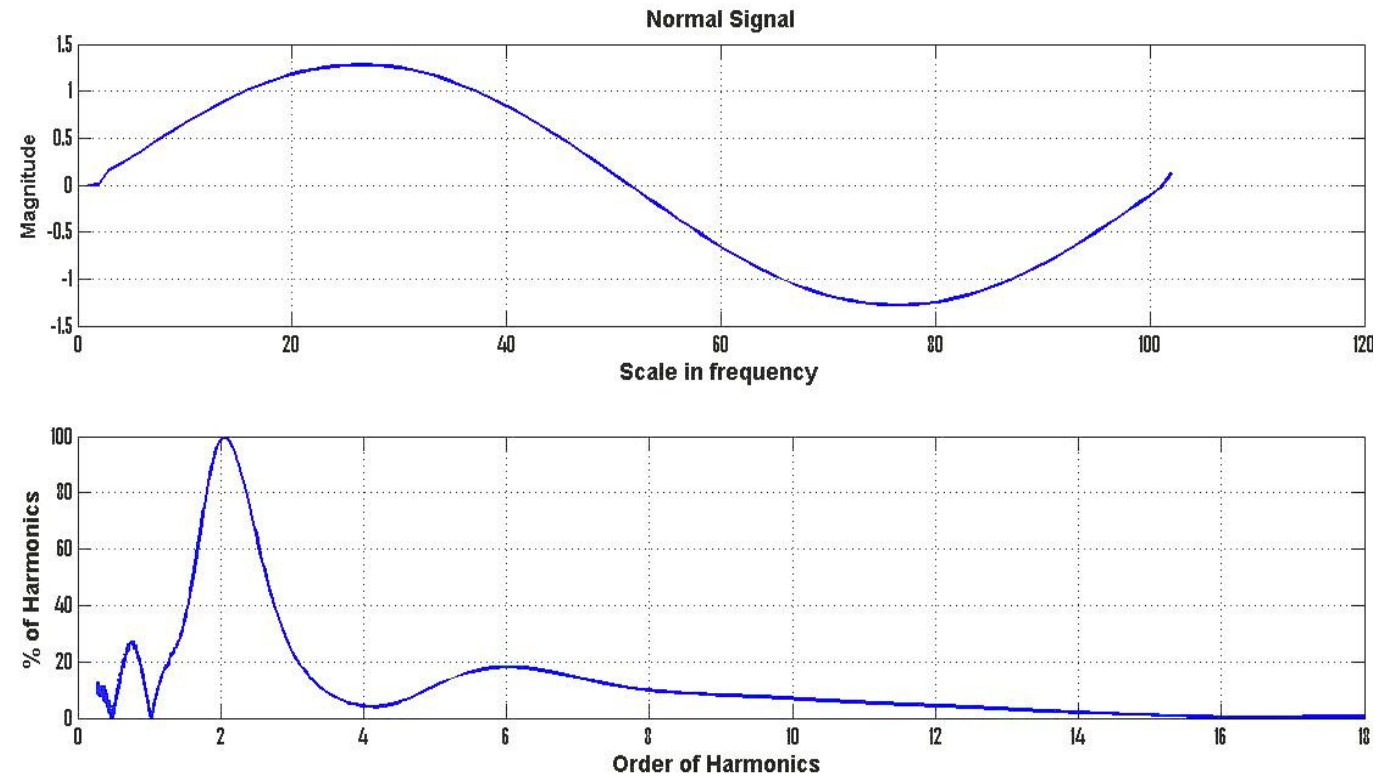

Fig.9 Frequency spectrum of MFCL current at faulty state by Wavelet 

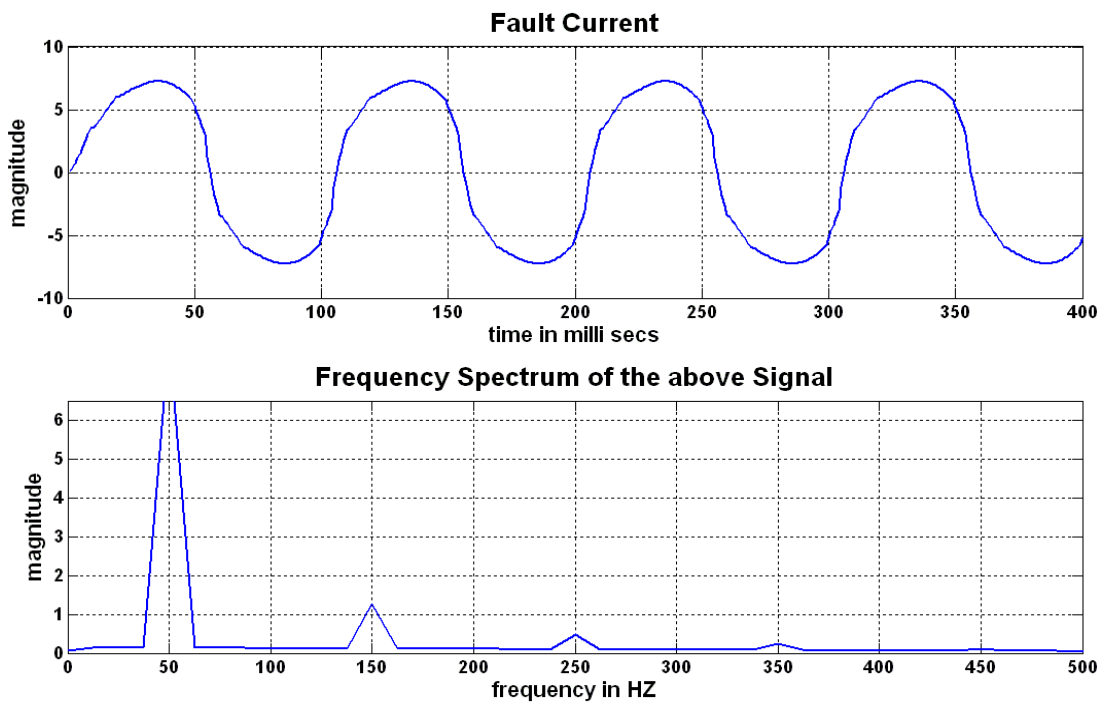

Fig.10 Frequency spectrum of MFCL current at faulty state by FFT

Fig $9 \& 10$ shows the frequency spectrum of MFCL current signal at faulty state by WT and FFT respectively.
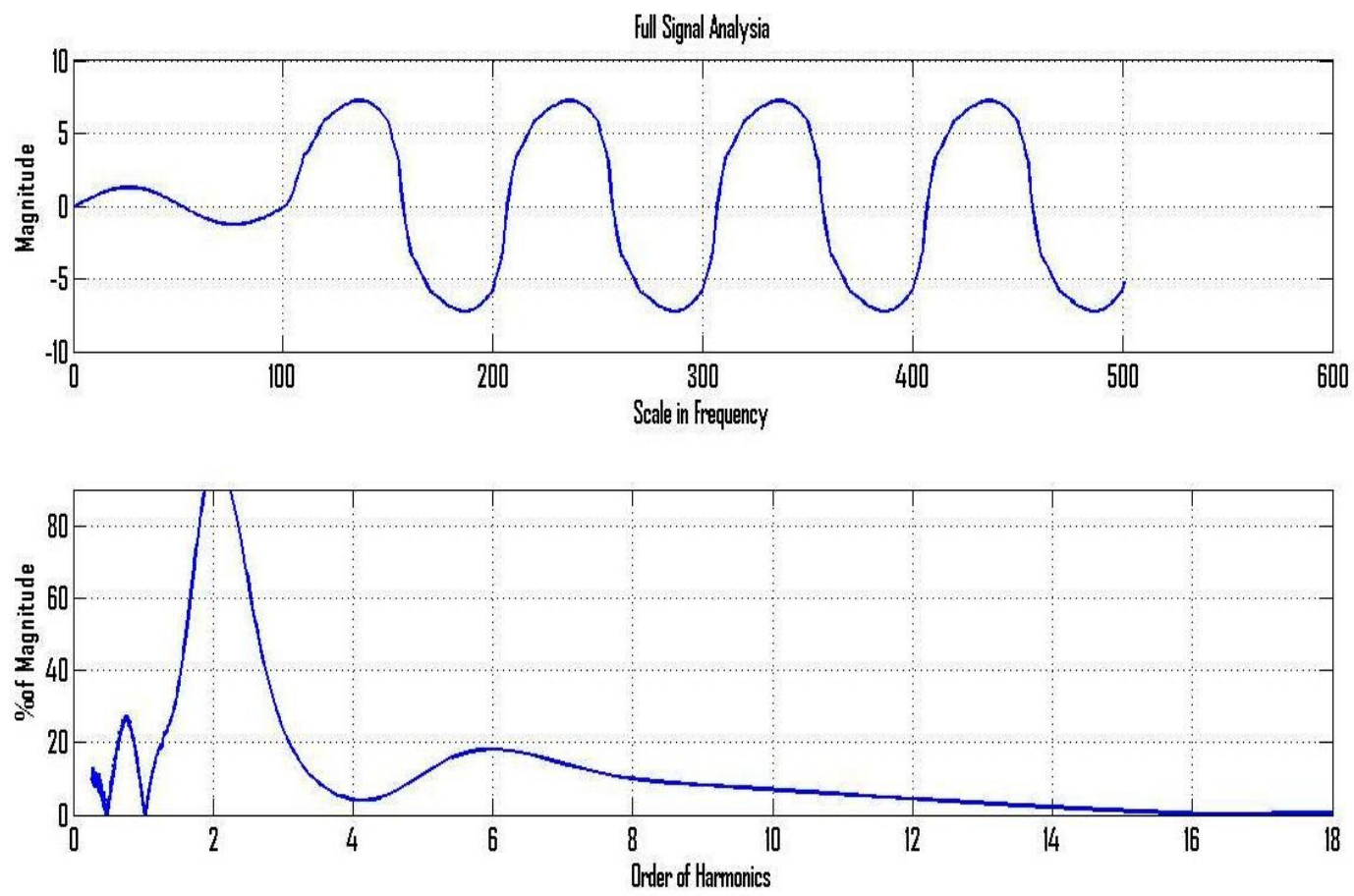

Fig.11. Frequency spectrum of MFCL Full current signal at normal andfaulty bothstate by Wavelet 
Table-3: Harmonics and Interharmonics in MFCL current at fault

\begin{tabular}{|c|c|c|}
\hline $\begin{array}{c}\text { Frequency } \\
(\mathrm{Hz})\end{array}$ & Order & Magnitude (\%) \\
\hline 25 & 0.5 & 13 \\
\hline 150 & 3.0 & 8.0 \\
\hline 175 & 3.5 & 6.0 \\
\hline 200 & 4.0 & 4.5 \\
\hline 250 & 5.0 & 4.0 \\
\hline 275 & 5.5 & 3.6 \\
\hline 350 & 7.0 & 2.8 \\
\hline
\end{tabular}

From Table-3 it is evident that at faulty state some amount of higher order harmonics and interharmonics (mainly $3^{\text {rd }}$, 5th and $7^{\text {th }}$ ) induced in the FCL current signal. But their magnitude is very less. The FCL current composed with mainly $1^{\text {st }}$ harmonic component

\section{Conclusions}

This paper has investigated the problems associated with the harmonics generation in fault current limiter. A prototype laboratory model and simulation were developed to observe the performance of MFCL. We observe that almost all power electronics and solid-state devices inject harmonics to supply system, which in turn degrades the quality of power. On the contrary the passive magnetic fault current limiter did not generate any significant harmonics in normal state (pre-fault). Under fault condition (post-fault) some amount of harmonics were generated but they were not rich in magnitude at all. In magnetic fault current limiter harmonics are generated due to irregular flux distribution in core in faulty state. Again irregular flux distribution occurs due to transition of MFCL transfer characteristic from saturated region to unsaturated region. So some amount of harmonics will be there in the network due to presence.

\section{REFERENCES}

[1] S. C. Mukhopadhyay, F. P. Dawson, M. Iwahara, and S. Yamada, "Analysis, design and experimental results for a passive current limiting device, IEE Proc.-Electr. Power Appl., vol. 146, no. 3, pp. 309316, May 1999.

[2] M. Iwahara, S. C. Mukhopadhyay, S. Yamada, and F. P. Dawson, "A full passive fault current limiter using the permanent magnet for the burden critical equipments," in J. Nonlinear Electromagnetic Systems: IOS Press, 1998, vol. 13, pp. 349-352.

[3] M. Iwahara and E. Miyazawa, "A numerical method for calculation of electromagnetic circuits using the tableau approach," IEEE TransMagn. Vol. 19, pp. 2457-2459, Nov. 1983.

[4] IwaharaM., Yamada S., Dawson F. P., FillionG., "A Passive Current Limiter for Power Semiconductor Protection," Industrial Applications Society Conference Proceeding, San Diego, 1996, Vol. 111,pp. 1298-1 301

[5] M. M. R. Ahmed,Ghanim A. Putrus, Li Ran and Lejun Xiao, "Harmonic Analysis and Improvement of a New Solid-State Fault Current Limiter" IEEE transaction on industry applications, vol. 40,No.4, July/August 2004

[6] Kit Po Wong, Van Long Pham “ Analysing Power system wave-forms using Wavelet-Transform approach" Proceedings of the 5th International Conference on Advances in Power System Control, Operation and Management, APSCOM 2000, Hong Kong, October 2000.

[7] A.B.Choudhury ,D.Roy, and M.Iwahara,'Field Distribution and Perfmance Analysis of a Passive Magnetic Fault-current Limiter under Transient Conditions" Electric Power Corponents and System, 37:pp-1195-1207,2009, 
[8] A.B.Choudhury ,D.Roy, M.Iwahara and S. Yamada "Harmonics and Interharmonics Estimation of a Passive Magnetic Fault Current Limiter Using Wavelet Transform" Jarnal of the Japan Society of Applied Electromagnetics and Mechanics, vol.17.No.4(2009),pp-606-610

\section{AUTHORS}

RajuPatwary graduated in Electrical Engineering from Haldia Institute of Technology in 2009 and obtained the M.E degree with specialization of Power System from Bengal Engineering and Science University, Shibpur in 2011. He is an Assistant Professor in Department of Electrical Engineering of Gargi Memorial Institute of Technology Balarampur, Baruipur, 24Parganas (S). He has 2 years teaching experience.

Pratyush Das graduated in Electrical and Electronics Engineering from Bengal College of Engineering and Technology, Durgapur and obtained the M.E degree with specialization of Power System from Bengal Engineering and Science University, Shibpur in 2011. He is an Assistant Professor in Department of Electrical Engineering of Global Institute of Management And Technology, Krishnagar, Nadia, West Bengal
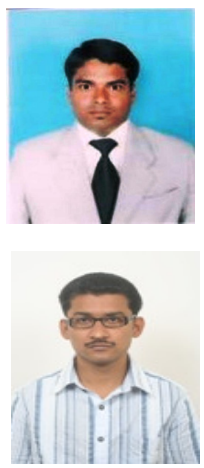